\title{
Behavioral modulation of stimulus-evoked oscillations in barrel cortex of alert rats
}

\author{
Subramaniam Venkatraman ${ }^{1}$ and Jose M. Carmena ${ }^{1,2,3 *}$ \\ Department of Electrical Engineering and Computer Sciences, University of California, Berkeley, CA, USA \\ 2 Helen Wills Neuroscience Institute, University of California, Berkeley, CA, USA \\ 3 Program in Cognitive Science, University of California, Berkeley, CA, USA
}

\section{Edited by:}

Rui M. Costa, Champalimaud

Neuroscience Programme, Instituto

Gulbenkian de Ciência, Portugal

\section{Reviewed by:}

Donald B. Katz, Brandeis University, USA

Joe Paton, Columbia University, USA

*Correspondence:

Jose M. Carmena, Department of EECS, University of California Berkeley, 517 Cory Hall, MC\# 1770, Berkeley, CA 94720, USA.

e-mail:carmena@eecs.berkeley.edu
Stimulus-evoked oscillations have been observed in the visual, auditory, olfactory and somatosensory systems. To further our understanding of these oscillations, it is essential to study their occurrence and behavioral modulation in alert, awake animals. Here we show that microstimulation in barrel cortex of alert rats evokes $15-18 \mathrm{~Hz}$ oscillations that are strongly modulated by motor behavior. In freely whisking rats, we found that the power of the microstimulation-evoked oscillation in the local field potential was inversely correlated to the strength of whisking. This relationship was also present in rats performing a stimulus detection task suggesting that the effect was not due to sleep or drowsiness. Further, we present a computational model of the thalamocortical loop which recreates the observed phenomenon and predicts some of its underlying causes. These findings demonstrate that stimulus-evoked oscillations are strongly influenced by motor modulation of afferent somatosensory circuits.

Keywords: evoked oscillations, thalamocortical, cortical microstimulation, behavioral modulation

\section{INTRODUCTION}

Since their original description (Bartley and Bishop, 1932; Chang, 1949), stimulus-evoked neuronal oscillations have been intensely studied. In the visual cortex, non stimulus-locked (induced) oscillations in the gamma range $(30-60 \mathrm{~Hz}$ ) (Friedman-Hill et al., 2000; Gray and Singer, 1989; Jagadeesh et al., 1992) and 5-8 Hz stimuluslocked (evoked) oscillations have been observed (Dinse et al., 1997). In the auditory system, tone and click-evoked oscillations in the 10-12 Hz range have long been observed (Galambos et al., 1952; Sally and Kelly, 1988) but are thought to occur only in anesthetized animals (Cotillon-Williams and Edeline, 2003). In the somatosensory system, whisker deflection evoked oscillations in the $10-15 \mathrm{~Hz}$ range have been observed in anesthetized animals (Ahissar et al., 2003; Muthuswamy et al., 1999) and during early development in awake animals (Khazipov et al., 2004). Spontaneous and odor induced oscillations in the olfactory system are also widely studied phenomena (Freeman, 2000; Delaney et al., 1994).

Cortical microstimulation in sensory areas is known to elicit an oscillatory response in both anesthetized and awake animals (Contreras and Steriade, 1996; Contreras et al., 1997). These oscillations are similar to the tone-evoked oscillations observed in the auditory system and their thalamic origin has been well established (Contreras and Steriade, 1996; Cotillon and Edeline, 2000). While the occurrence of stimulus-evoked oscillations in anesthetized animals has been well documented, experiments on awake subjects have yielded conflicting results (Cotillon-Williams and Edeline, 2004). To shed light on the functional significance of these oscillations, it is essential to study their occurrence and behavioral modulation in alert, awake animals.

Modulation of afferent sensory transmission by active movement is a well known phenomenon (Shin and Chapin, 1990). The rodent vibrissa system provides an excellent model to study this modulation. During quiet immobility, whisker deflections evoke large-amplitude, highly distributed cortical sensory responses (Castro-Alamancos and Oldford, 2002; Fanselow and Nicolelis, 1999; Ferezou et al., 2007; Hentschke et al., 2006; Lee et al., 2008; Scaglione and Moxon, 2006). Whereas, during active whisking, passive whisker deflections evoke small-amplitude localized sensory responses. Hence sensory responses in the rat whisker system are thought to be dynamically modulated by motor behavior.

It is believed that this may function as a sensitive detection system (a wake-up call), alerting the animal to unexpected sensory inputs (Ferezou et al., 2007). We show that this effect can be recapitulated by replacing sensory stimulation with cortical microstimulation. The neural response to microstimulation can be considered to be the impulse response function of the thalamocortical loop (Freeman, 2000). Thus studying microstimulation evoked neural responses presents an excellent method to explore modulation of the thalamocortical loop by behavioral state.

Further, we explore whether cortical microstimulation evoked oscillations in the rat somatosensory system are also modulated by motor behavior. Similar modulation to that observed in sensory responses would suggest that both phenomena are brought about by common or related underlying causes. It would also provide an experimental link between stimulus-evoked oscillations and modulation of afferent somatosensory circuits by motor behavior.

\section{MATERIALS AND METHODS ANIMALS}

Five adult female Sprague-Dawley rats weighing 200-300 g were used in this study. They were initially handled for 1 week and trained to sit calmly while restrained in a cloth bag and body restrained in a semi-cylindrical tube. Once rats learned to sit quietly, they were implanted with microwire arrays. All animal procedures conformed 
to the NIH and USDA regulations and were approved by the UC Berkeley Animal Care and Use Committee.

\section{SURGICAL PROCEDURE}

Microwire arrays with two $35 \mu \mathrm{m}$ diameter Platinum/Iridium stimulating electrodes (CD Technologies, Durham, NC, USA) and 16 tungsten recording electrodes ( $35 \mu \mathrm{m}$ diameter, $8 \times 2$ array) were implanted in barrel cortex. Rats were anaesthetized with ketamine-xylazine supplemented with isoflurane gas anesthesia. After a craniotomy and durotomy, the electrode array was stereotactically inserted into the infragranular layer of the primary somatosensory barrel field (S1bf) in one hemisphere. Stereotaxic coordinates relative to bregma were used to center the arrays in S1bf [anteroposterior (AP), $-3.0 \mathrm{~mm}$; mediolateral (ML), $+5.5 \mathrm{~mm}$; dorsoventral (DV), $-1.2 \mathrm{~mm}$ ]. The craniotomy was sealed with cyanoacrylate and the array was firmly attached to the skull using dental acrylic. Intraoperative recording of multiunit activity and post-operative receptive field mapping, using the MAP system (Plexon Inc, Dallas, TX, USA), were used to ensure that arrays were located in barrel cortex. Placement of electrodes in the infragranular layer was verified by comparing the polarity of observed spontaneous oscillations to known depth profiles of oscillations (Kandel and Buzsaki, 1997).

\section{CORTICAL MICROSTIMULATION}

Biphasic stimulating pulses were delivered through bipolar 200$500 \mathrm{k} \Omega$ platinum/iridium microelectrodes (AM Systems pulse generator and stimulus isolation unit, Sequim, WA, USA). Pulse length was set to $250 \mu$ s and the stimulation typically consisted of two pulses of $10-20 \mu \mathrm{A}$ separated by $3 \mathrm{~ms}$. Microstimulation was delivered while rats were restrained and the interval between pulses was varied in a pseudo-random manner between 1-5 s.

\section{WHISKER TRACKING}

A full description of the real-time video tracking and closed-loop microstimulation system used in this work has been published previously (Venkatraman et al., 2009). In brief, rats were body restrained and one whisker was tagged using a light self-adhesive foam marker. We did not head-fix rats since this led to increased signs of stress and significantly reduced instances of spontaneous whisking. The video tracking system captured video at 100 frames/s using an EC-640C camera (Prosilica, Burnaby, BC, Canada) placed above the rat and used custom software to track the whisker marker.

\section{CORRELATION ANALYSIS}

We defined the power of the evoked neural oscillation as the power in the $10-20 \mathrm{~Hz}$ band of the local field potential (LFP) $100-500 \mathrm{~ms}$ post-microstimulation. The strength of whisking was defined as the power in the $5-20 \mathrm{~Hz}$ band of the recorded whisker trace $0-100 \mathrm{~ms}$ pre-stimulation. The correlation between these two metrics was defined as the correlation between the power of the evoked oscillation and the strength of whisking.

It is conceivable that state dependent evoked responses could occur if rats were 'quiet' for the first half of the trials and awake and 'whisking' for the next half. To demonstrate that rats showed rapid transitions between behavioral states, we calculated the correlation between evoked neural oscillations and whisking strength at some time lag ( $t$ seconds, $t<1$ ). If behavioral states changed on the order of tens of seconds, one would expect that whisking strength ' $t$ ' seconds prior to stimulation would be highly correlated to the strength of stimulus evoked oscillations. However, if behavioral states changed rapidly, one would expect that this correlation would drop off rapidly with increasing magnitude of $' t$ '

\section{RESULTS BEHAVIORAL MODULATION}

Neural responses to cortical microstimulation have been studied by several researchers (reviewed in Tehovnik et al., 2006). Cortical microstimulation typically triggers nearby neurons to fire spikes immediately after stimulation. This is followed by a prolonged period $(\sim 100 \mathrm{~ms})$ of decreased firing rate (mediated by inhibitory circuits (Butovas et al., 2006) followed by a short rebound excitation and return to baseline firing rate (Butovas and Schwarz, 2003). We observed a similar neural response to microstimulation in awake, freely roaming rats (Venkatraman et al., 2009). However we also noticed a fraction of trials where this response was followed by oscillations phase-locked to the stimulation.

To investigate whether the variability in evoked oscillations was related to motor activity in the whisker system, we tracked the position of a single whisker in awake rats while microstimulating at random intervals $(1-5 \mathrm{~s})$. The neural response to microstimulation when rats were actively whisking showed a small initial inhibition and no subsequent oscillations (Figure 1A). In contrast, we found that when rats were quietly immobile, the LFP response to microstimulation consisted of $\sim 15 \mathrm{~Hz}$ oscillations following the initial prolonged inhibition (Figure 1B). The multiunit activity showed burst firing coherent with the negative deflections of the LFP.

We observed a significant negative correlation between the power of the evoked LFP oscillation and the strength of whisking $(R=-0.50, P<0.001$; Figure 2). This is further illustrated in Figure 3 where 50 trials with lowest whisking strength were classified as 'Quiet' and 50 trials with the highest whisking strength were classified as 'Whisking. The raster and average LFP response when 'Quiet' show evoked oscillations in response to cortical microstimulation (Figure 3A). We calculated the power spectral density of the LFP response during time $100-500 \mathrm{~ms}$ post-microstimulation in each trial. The average of all such power spectral densities shows a peak at $17 \mathrm{~Hz}$ (Figure 3B). The initial multiunit response to cortical microstimulation (2-10 ms post stimulation in Figure 3C) in 'Quiet' trials was statistically similar to 'Whisking' trials (considering 15 multiunits from the same animal, paired Student's $t$-test, $P>0.25$ ) suggesting that cortical excitability is not modulated by behavior. However, microstimulation in 'Quiet' trials evoked a stronger inhibition (comparing LFP 20-100 ms after stimulation, Mann-Whitney two-tailed test, $P<0.001)$ and more pronounced evoked oscillations (comparing power spectral density of LFP $12-18 \mathrm{~Hz}$, Mann-Whitney two-tailed test, $P<0.001$ ). $94 \%$ of the evoked responses in 'Quiet' trials showed higher spectral power in the $12-18 \mathrm{~Hz}$ band compared to the average power in whisking trials showing that oscillations were reliably evoked in quiet trials. These analyses show that the neural response to microstimulation is strongly dependent on the behavioral state of the animal. Similar behavioral modulation of neural responses 


\section{A}

LFP 1

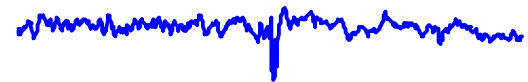

LFP 2

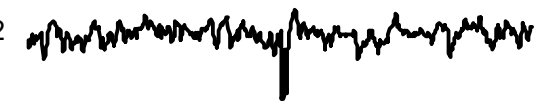

Spike 1

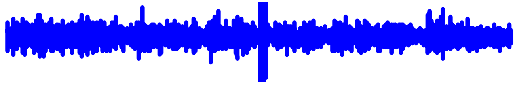

Spike 2
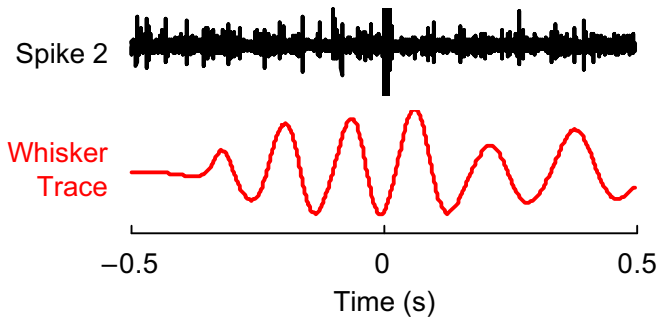

B
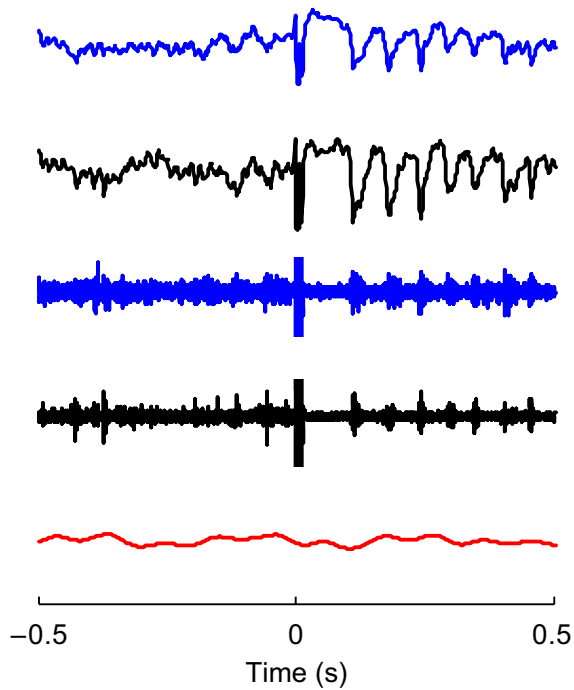

FIGURE 1 | Evoked responses to cortical microstimulation. (A) $1-200 \mathrm{~Hz}$ local field potential (LFP) and 0.5-10 kHz multiunit activity (Spike) recorded from two electrodes in infragranular layer of barrel cortex of awake rats are shown along with traces of whisker movements. Microstimulation (at $0 \mathrm{~s}$ ) delivered during active whisking typically induced a small neural response which is partially obscured by the stimulus artifact at $0 \mathrm{~s}$. (B) On the other hand, microstimulation delivered during periods of no whisker movement typically induced a long period of reduced neural activity followed by a series of $15-18 \mathrm{~Hz}$ rhythmic oscillations in the LFP and concomitant spike bursts in multiunit recordings.
A

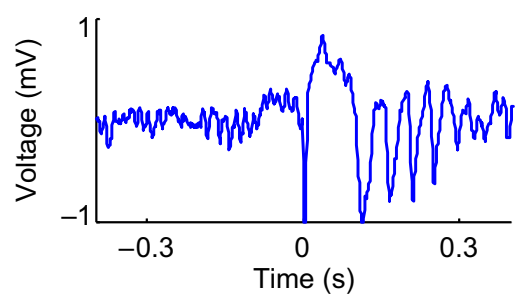

B

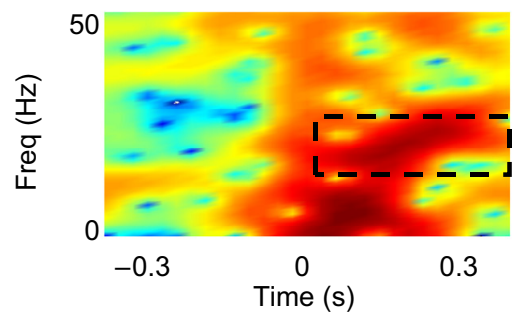

C

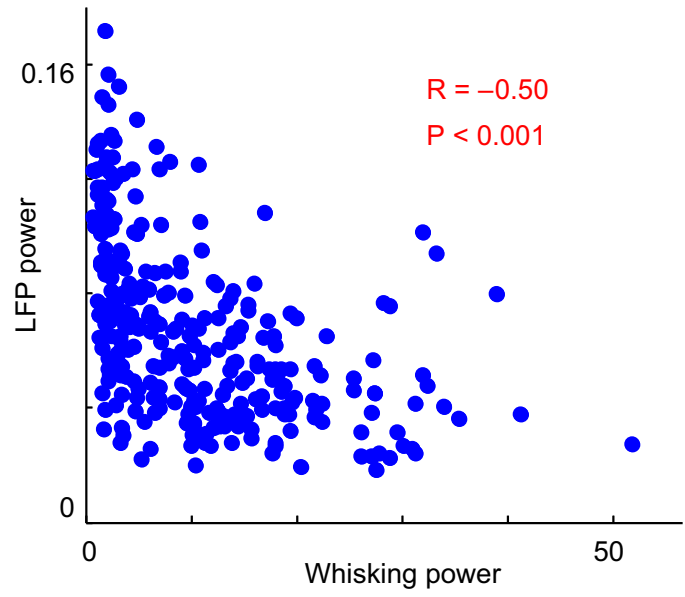

FIGURE 2 | Correlation between whisking and evoked LFP oscillations. (A) Example LFP trace of microstimulation evoked oscillations. (B) Spectrogram of above LFP trace. The power in the 10-20 Hz band from 100-500 ms after microstimulation is used as a metric of the power of the evoked LFP oscillation (dashed black box). (C) Scatter plot shows an inverse relation between the power of the evoked LFP oscillations and the strength of whisking in 300 stimulations on one rat.

was observed in a total of five rats (Figures 3,5 and $\mathbf{6}$ are obtained from data from three different rats). It should be noted that there was no explicit sign of bodily activity such as whisker movement or twitching correlated with the evoked oscillations. Further, the evoked oscillations died down within $500 \mathrm{~ms}$ and never resulted in kindled seizures.

\section{CAUSE OF MODULATION}

It is conceivable that behavioral modulation of evoked responses could be caused by the state of alertness or arousal of the animal. It is well known that the state of arousal of the animal modulates the response properties of the thalamocortical network (as reviewed in Castro-Alamancos, 2004; McCormick and Bal, 1997). To explore 

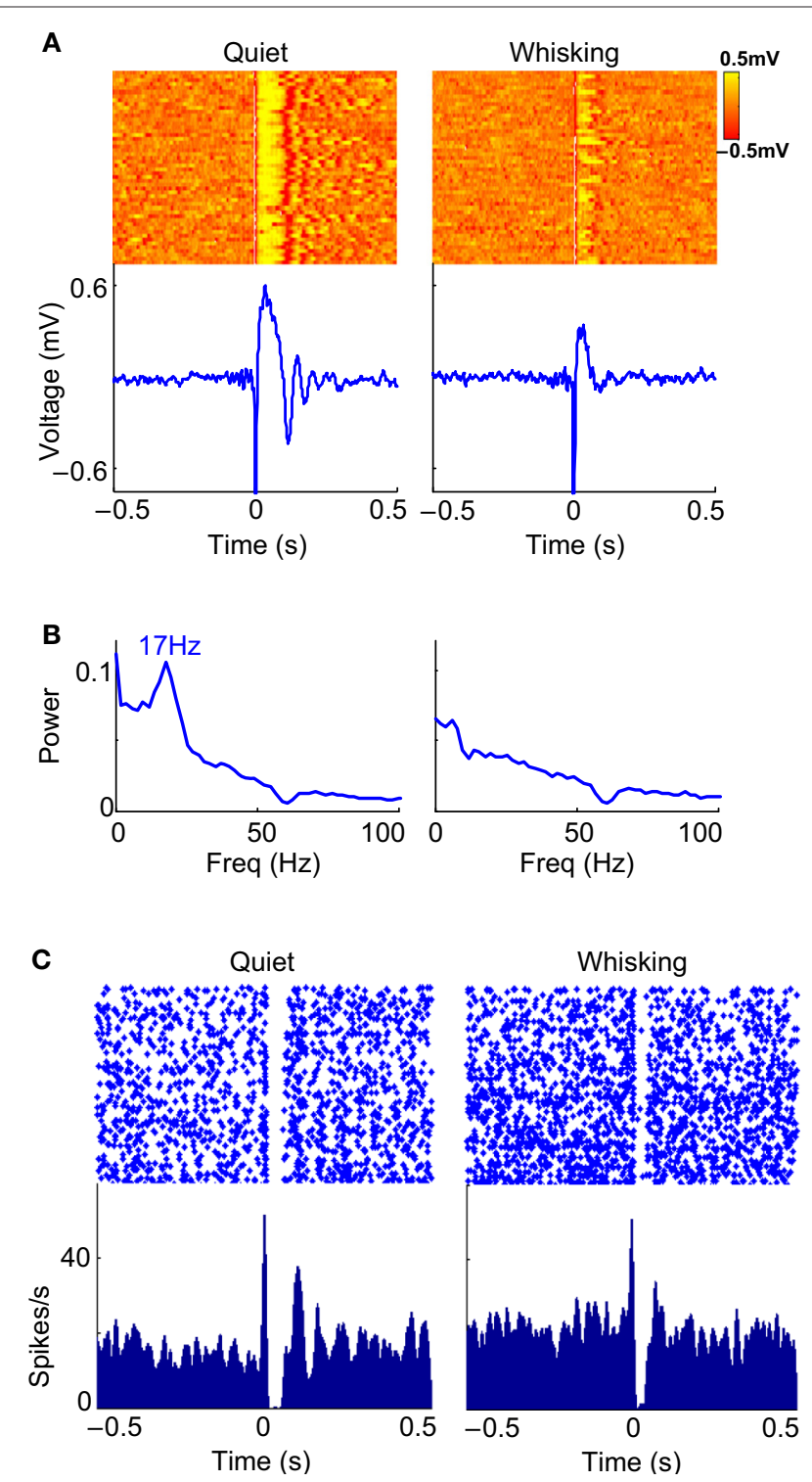

FIGURE 3 | LFP and multiunit evoked responses. (A) Raster plot and average evoked LFP responses to cortical microstimulation (at $0 \mathrm{~s}$ ) in 'whisking' and 'quiet' trials. (B) The average power spectral density of the LFP during the period 100-500 ms after microstimulation in whisking and quiet trials. (C) Raster and histogram of evoked multiunit responses to

microstimulation during whisking and quiet trials. The quiet trials show a lower baseline firing rate, a prolonged inhibition and oscillatory bursting but the initial excitation (0-5 ms) is similar to that observed when whisking.

whether level of alertness played a role in our experimental setup, we measured the power in the delta frequency band $(1-4 \mathrm{~Hz})$ of the LFP which is often used as a marker of alertness (Hentschke et al., 2006). We found that the power of the microstimulationevoked oscillations was not significantly correlated to $0-500 \mathrm{~ms}$ pre-stimulus delta power $(R=0.02, P>0.5)$. This suggests that level of alertness were not an influencing factor in the observed modulation.

Another potential source of modulation could exist if rats fell asleep when quiet. To rule out this possibility, we examined

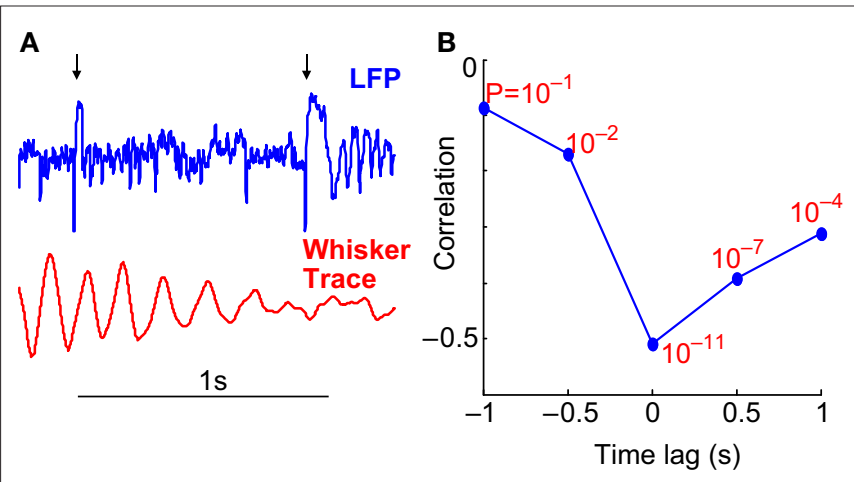

FIGURE 4 |Time constant of behavioral states. (A) Example of whisking trace and LFP responses to two closely spaced microstimulations. The time of microstimulation is indicated using black arrows. The significant difference in LFP response shows that evoked responses to microstimulation are rapidly modulated by changes in motor behavior. (B) The correlation between LFP oscillation power and whisking strength is plotted at different time lags with the correlation $(R)$ on the $Y$-axis and the ' $P$ ' value of each correlation indicated in red. Note that the correlations are negative with the highest magnitude of correlation $(-0.50)$ occurring at 0 lag. The rapid drop-off in the correlation implies rapid switching of behavioral states.

occasions where the neural response to microstimulation was significantly different on closely spaced stimulations (Figure 4A). To demonstrate that rats showed rapid transitions between behavioral states, we calculated the correlation between evoked neural oscillations and whisking strength ' $t$ ' seconds before or after stimulation. This analysis reveals to what degree whisker movements more distant in time from the microstimulation influenced the evoked oscillations. If behavioral states changed slowly, one would expect that whisking strength ' $t$ ' seconds prior to stimulation would be strongly correlated to the strength of stimulus evoked oscillations. We found that the correlation dropped off rapidly with a time lag as low as $500 \mathrm{~ms}$ (Figure 4B) implying rapid transitions between behavioral states. Such rapid transitions (Hentschke et al., 2006) are incongruent with sleep and more likely caused by rapid changes in motor behavior.

To further ensure that animals were awake and alert, we trained two rats on a variable interval tone detection task while restrained. A tone was played after random intervals and rats learned to wait and respond to the tone with a lick within $1 \mathrm{~s}$. We also delivered cortical microstimulation randomly $0.5-1 \mathrm{~s}$ before the occurrence of the tone. During this period, rats would sometimes sit in a quiet immobile state and sometimes actively whisk. This microstimulation did not have any relevance to the behavioral task but we could now analyze the neural response to microstimulation considering only those trials where rats responded within $1 \mathrm{~s}$ after the tone. We repeated the analysis of Figure 2 on this dataset and found very similar results (Figure 5). The reaction time on the task was in fact lower when the rats were quiet than when they were whisking (Median response time when quiet $=0.30 \mathrm{~s}$ and whisking $=0.56 \mathrm{~s}$, Mann-Whitney two-tailed test, $P<0.001)$ suggesting that the rats were equally if not more alert when quiet. Taken together, these results argue against the possibility that alertness, arousal or sleep play a role in the behavioral modulation observed in this work. 


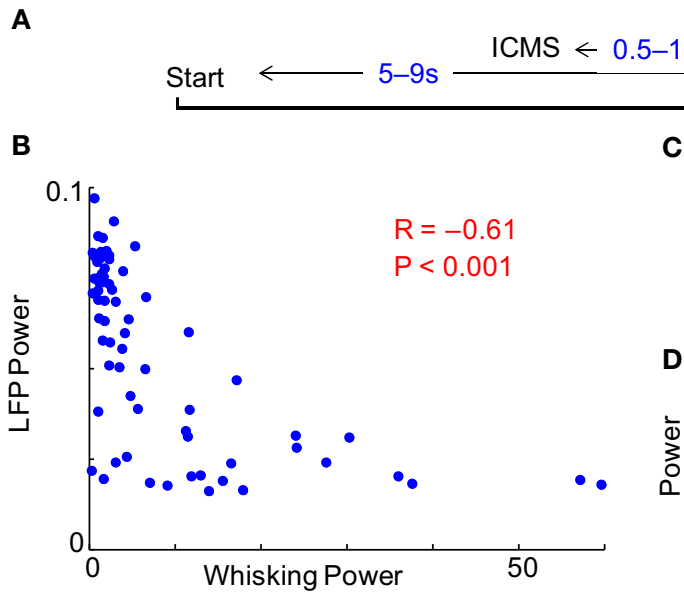

FIGURE 5 | Behavioral modulation in alert rats. (A) Structure of the variable interval tone detection task on which rats were trained. Cortical microstimulation was introduced $0.5-1 \mathrm{~s}$ before the tone stimulus on some trials and had no relevance to the task. (B) The inverse relation
This leads us to the conclusion that the observed effect is likely caused by motor modulation of the somatosensory thalamocortical loop. It should be noted that there exist points in the lower left hand corner of Figures 2C and 5B demonstrating that the relationship between whisking strength and evoked oscillations in not purely linear. This suggests that although motor behavior is a dominant modulator of the somatosensory thalamocortical loop, other causes of modulation (like attention) exist.

We then investigated the effect of varying the microstimulation parameters on the evoked neural response. Increasing the amplitude of stimulation led to oscillatory evoked responses even in actively whisking rats (Figure 6). The neural response to $10 \mu \mathrm{A}$ stimulation (Figure 6A) was strongly modulated by behavior whereas $30 \mu \mathrm{A}$ stimulation (Figure 6B) evoked oscillations irrespective of behavioral state. This is also evidenced in the reduction of the correlation between oscillation power and whisking strength in $30 \mu \mathrm{A}$ stimulation. This suggests that a strong volley of spikes can evoke oscillations even when the network is in the 'tonic' state. The increase in intensity of stimulation increased the amplitude of the evoked oscillations but did not alter the frequency. This is consistent with previous experiments performed under anesthesia (Contreras and Steriade, 1996).

\section{RELATION TO SPONTANEOUS RHYTHMS}

In the rat somatosensory system, two prominent $<20 \mathrm{~Hz}$ oscillations have been observed. Sleep spindles are $7-14 \mathrm{~Hz}$ oscillations observed in early stages of sleep that wax and wane over a period of 1-3 s (Steriade et al., 1990). Spontaneous spindles are also observed under ketamine-xylazine anesthesia but only show a waning phase (Contreras and Steriade, 1996). Cortical microstimulation evoked oscillations appear very similar to spontaneous ketamine spindles (Figure 7A). This agrees with the hypothesis that spindles are often initiated by a naturally occurring synchronous volley of spikes from the cortex (Fuentealba et al., 2004). The corticothalamic nature of spontaneous ketamine spindles and cortical microstimulation evoked oscillations has been well established (Contreras and Steriade, 1996; Fuentealba et al., 2004).

The second prominent oscillations occur in the $8-10 \mathrm{~Hz}$ band and are known to spontaneously occur in quiet immobile rats. They have been variously called high voltage rhythmic spikes (HVRS) (Shaw, 2007), high voltage spike-and-wave spindles (HVSs) (Kandel and Buzsaki, 1997), Mu Rhythms (Fontanini and Katz, 2005; Nicolelis and Fanselow, 2002) and Spike Wave Discharges (SWDs) (Kelly, 2004). An example of such an oscillation in an awake rat is shown in Figure 7B and its average power spectral density is shown in Figure 7C. This figure clearly demonstrates that SWDs have a different frequency range compared to the observed microstimulation evoked oscillations. It should also be noted that the pre-stimulus period of Figures 3 and 5 do not show any prominent LFP oscillations. We discarded the few stimuli which occurred during ongoing $8-10 \mathrm{~Hz}$ oscillations to ensure that they did not play a role in the observed effects.

\section{MODELING}

A number of detailed computational models of thalamic and thalamo-cortical networks have been developed (Destexhe et al., 1993; Wang et al., 1995) which provide insight into some of the basic neuronal mechanisms underlying thalamocortical oscillations. We constructed a population model in MATLAB (MathWorks Inc., Natick, MA, USA) to explore the possible origins of the evoked neural oscillations in response to cortical microstimulation. This model was based on the one developed in (Suffczynski et al., 2004) to explore the transitions between spontaneous spindle oscillations and SWDs. We extended this model to better account for the effect of modulatory neurotransmitters in the thalamus by modeling the $\mathrm{Na}^{+}$and $\mathrm{K}^{+}$leak currents $\left(g_{\text {Na leak }}\right.$ and $\left.g_{\text {Kleak }}\right)$. This model recreates patterns of evoked oscillations allowing us to infer what properties are necessary and sufficient to account for the observed phenomena.

The model consists of four sub-parts modeling thalamic relay cells (TC), reticular thalamic cells (RE), pyramidal neurons in the cortex 
A

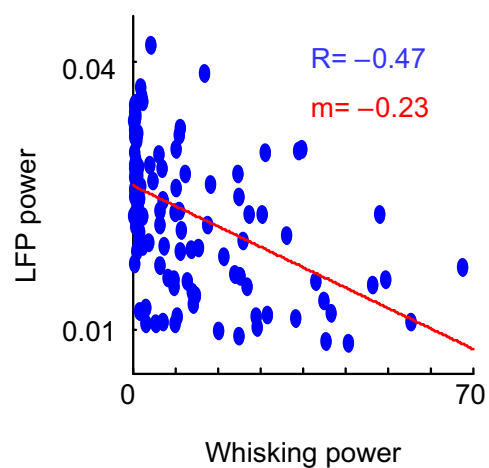

B

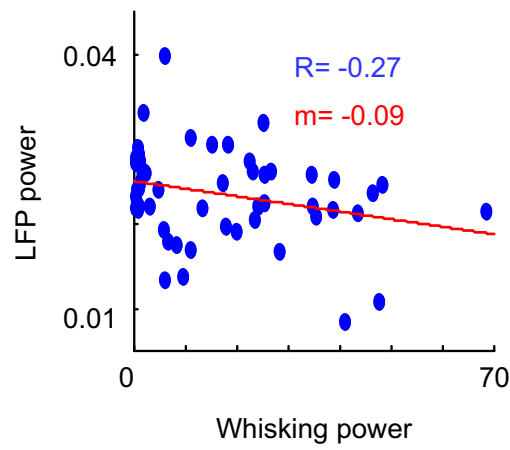

FIGURE 6 |Amplitude of stimulation. (A) Scatter plot, raster and average of the LFP response to cortical microstimulation at $10 \mu \mathrm{A}$. The correlation coefficient between whisking strength and strength of evoked oscillations is
$10 \mu \mathrm{A}$ Stimulation

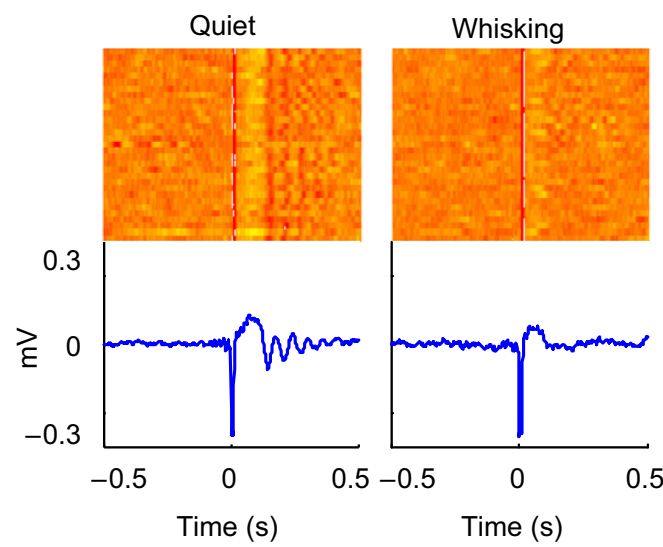

$30 \mu \mathrm{A}$ Stimulation

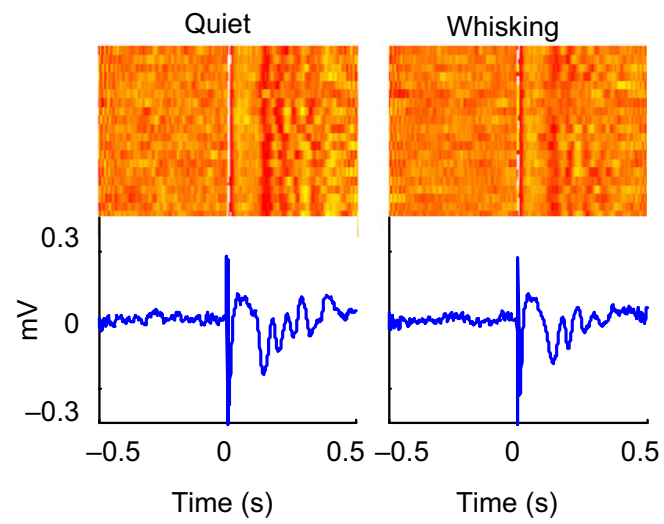

denoted by ' $R$ ' ( $P<0.05$ in both cases) and slope of the linear fit of the scatter plot is denoted by ' $m$ '. (B) Neural response to microstimulation at $30 \mu \mathrm{A}$ shows evoked oscillations irrespective of behavioral state.

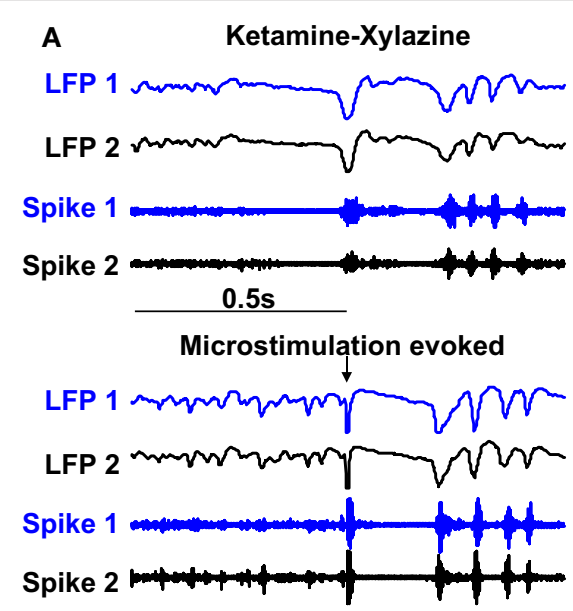

FIGURE 7 | Comparison to spontaneous oscillations. (A) Spontaneous waning oscillations in LFP and multiunit recordings while under

ketamine-xylazine anesthesia (ketamine spindles) are very similar to

microstimulation evoked responses in the same animal. (B) Spontaneous
B Mu Rhythm

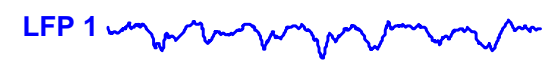

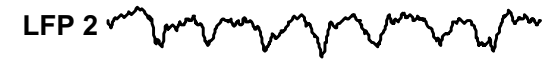

Spike 1

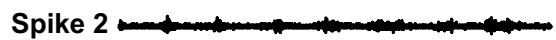

Whisker

Trace

$0.5 \mathrm{~s}$

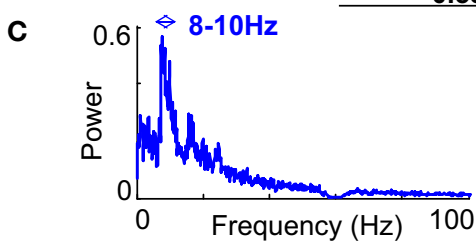

oscillations (SWDs) observed in awake immobile rats often accompanied by whisker twitching. (C) Average frequency spectrum of SWDs shows a peak at 8-10 Hz clearly different from that observed in microstimulation evoked oscillations. 


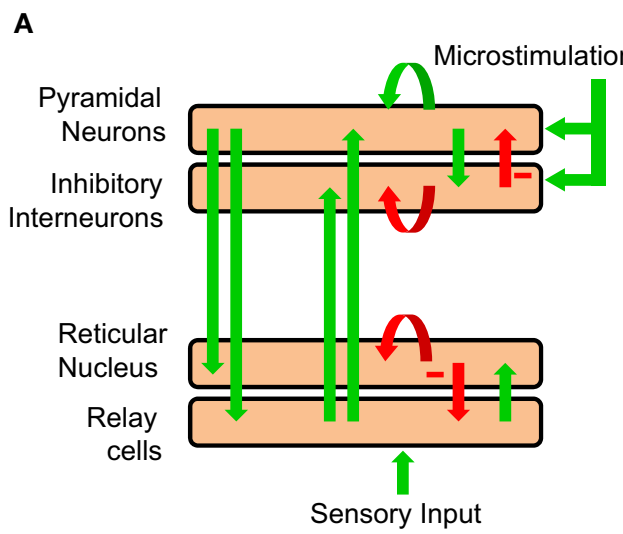

FIGURE 8 |Thalamocortical model. (A) Computational model of the thalamocortical loop used to investigate potential mechanisms of the experimentally observed evoked responses. The green arrows denote excitatory connections and the red arrows inhibitory. (B) Evoked LFP response of the thalamocortical model to a burst of spikes in the cortex (at $0 \mathrm{~s}$ ) shows a similar response to experimentally observed data. (C) The model suggests that a
B

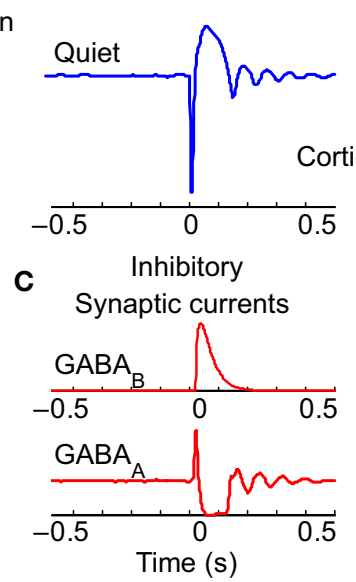

D

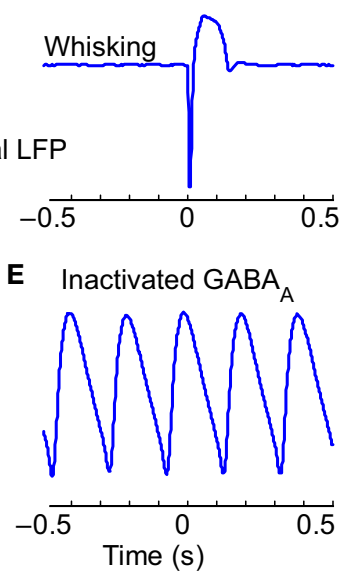

GABA $_{B}$ mediated IPSP in thalamocortical cells plays a significant role in the initial prolonged inhibition. A series of GABA mediated IPSPs in thalamic neurons along with their intrinsic bursting properties seem to be responsible for the oscillatory evoked response. (D) The evoked response in the modeled whisking state is similar to that experimentally seen. (E) Application of GABA antagonists induces lower frequency sustained oscillations in the thalamocortical model.

(PY) and inhibitory interneurons in the cortex (IN) (Figure 8A). The transformation between mean membrane potential and firing rate in thalamic neurons takes into account the $I_{\mathrm{T}}$ current which underlies burst firing. Cortical microstimulation was modeled by the injection of a strong excitatory input on PY and IN neurons for $5 \mathrm{~ms}$. It was assumed (as in Suffczynski et al., 2004) that cortical LFP recordings show similar behavior to the negative of the mean membrane potential of the PY cell population. The equations governing the TC neurons are shown in detail in the Appendix.

The model recreates the evoked LFP oscillations in response to cortical microstimulation (Figure 8B). According to the model (Figure 8C), the initial burst of spikes in response to cortical microstimulation causes a prolonged hyperpolarization of neurons due to the activity of $\mathrm{GABA}_{\mathrm{B}}$ receptors in the thalamus and cortex. This is in agreement with (Butovas et al., 2006) and the fact that $\mathrm{GABA}_{\mathrm{B}}$ is typically activated only by a strong volley of spikes as created by cortical microstimulation. Prolonged hyperpolarizations of RE neurons are known to precede spontaneously occurring spindle oscillations (Fuentealba et al., 2004). This hyperpolarization likely deinactivates the $\mathrm{Ca}^{2+}$ dependent T-current $\left(I_{\mathrm{T}}\right)$ leading to a low threshold spike and rebound excitation. In a similar manner, the microstimulation evoked hyperpolarization sets off evoked oscillations in the model by a combination of GABA $_{\mathrm{A}}$ mediated IPSPs (Figure 8C) and the intrinsic bursting property of TC and RE neurons. The oscillations are further transferred to the cortex by the TC neurons.

The precise neuromodulatory mechanism by which motor activity modulates the somatosensory thalamocortical system is unknown. Potential modulatory neurotransmitters are acetylycholine, serotonin, norepinepherin etc which act primarily by modifying the potassium leak conductance $\left(g_{\mathrm{K} \text { leak }}\right)$ in thalamic and reticular neurons (McCornick, 1992). Moreover, the sensory input to thalamic neurons is expected to be higher during active whisking. Reducing $g_{\mathrm{K} \text { leak }}$ in RE and increasing sensory inputs led to depolarization of
RE neurons in the model. We found that the evoked response now showed a reduced inhibition and no oscillations in a manner similar to that observed in actively whisking rats (Figure 8D). Further, it is known that the administration of $\mathrm{GABA}_{\mathrm{A}}$ antagonists to the ferret LGN in vitro slowly perverts normal spindle waves into a highly synchronized slow oscillation similar to SWDs. Blocking of GABA in the model led to a similar result (Figure 8E).

This model suggests that the oscillatory properties of TC and RE neurons can account for the evoked oscillations and behavioral modulation seen in our experiments. The exact mechanisms underlying spindle initiation and synchronization are not completely understood (Fuentealba and Steriade, 2005). Future models of evoked thalamocortical oscillations should include the hyperpolarization-activated current $I_{\mathrm{h}}$ and the $\mathrm{Ca}^{2+}$-activated currents $I_{\mathrm{K}[\mathrm{Ca}]}$ and $I_{\mathrm{CAN}}$ which are thought to play a role in spontaneous spindles (Destexhe et al., 1993). Further, norepinephrine and serotonin are known to abolish spindle wave generation through an enhancement of $I_{\mathrm{h}}$ in TC neurons (Lee and McCormick, 1996) and this mechanism may contribute to behavioral modulation.

\section{DISCUSSION}

In this work, we have demonstrated that microstimulation-evoked oscillations in rat barrel cortex are strongly modulated by active whisking. This phenomenon is also seen in alert rats engaged in a challenging behavioral task, suggesting that the observed modulation is truly motor driven.

\section{STARTLE RESPONSE}

Modulation of the whisker sensory system by motor behavior has been observed by a number of researchers (Castro-Alamancos and Oldford, 2002; Fanselow and Nicolelis, 1999; Ferezou et al., 2007; Hentschke et al., 2006; Lee et al., 2008). During active whisker movements, somatosensory afferent circuits seem to act like a linear 
low-gain input stage to faithfully transmit patterns of spikes from the periphery. In contrast, during quiet immobility, the system seems to act like a non-linear high-gain input stage to optimally detect and magnify single stimuli. This may function as a sensitive detection system (a wake-up call), alerting the animal to unexpected sensory inputs (Ferezou et al., 2007). Moreover, this modulation persists after transaction of the infraorbital nerve (Hentschke et al., 2006) suggesting that it is not caused by afferent sensory signals and must be central in origin. This gating of sensory inputs is thought to occur at the level of the brainstem and the thalamus (Lee et al., 2008). Our findings show that the neural response to microstimulation in barrel cortex is also modulated by active whisking. This occurs in the form of modulation of the initial inhibition as well as the long-lasting evoked oscillations.

The question then arises as to whether whisker deflection also sets off the kind of oscillatory evoked responses seen in response to cortical microstimulation? Whisker deflection evoked oscillations at $16 \mathrm{~Hz}$ have been observed in anesthetized animals (Ahissar et al., 2003; Muthuswamy et al., 1999) but are typically not seen in awake, behaving rats. This could be due to a number of factors. Cortical microstimulation, even at low stimulus levels, might excite more thalamic spikes than caused due to physical whisker deflections. Further, it is known that stimulation of pre-thalamic neurons is less effective at setting off oscillations than cortical stimulation (Steriade et al., 1990). This is thought to be because cortical stimulation directly excites RE neurons whereas pre-thalamic afferent fibers and incoming sensory stimuli do not.

Intracellular recordings in RE neurons (Contreras and Steriade, 1996) have shown that cortical microstimulation triggered oscillations increase in amplitude as the cells are hyperpolarized from -54 to $-75 \mathrm{mV}$. The modulation of evoked oscillations by whisking of rats suggests that motor behavior rapidly depolarizes somatosensory reticular neurons. This modulation of membrane potential can be readily achieved by neurotransmitters like acetylcholine, serotonin, norepinephrine etc by varying the $\mathrm{K}^{+}$leak conductance. This mechanism potentially also plays a role in behavioral modulation of sensory evoked responses observed in the rat whisker system. This hypothesis can be verified in future experiments using patch clamp recordings of RE neurons in awake, behaving rodents.

\section{EVOKED OSCILLATIONS}

The functional role of stimulus-evoked oscillations has been the subject of much debate. It has been suggested that bursting, as seen in microstimulation-evoked responses, may provide better signal to noise and thus facilitate detection of a stimulus (Sherman, 2001). However, it has also been found that the thalamus is insensitive to external inputs during spindles and hence spindles are thought by some to represent a cutoff from the external world (Llinas and Steriade, 2006).

Sensory stimuli like tones and flashes of light also evoke oscillatory responses under certain circumstances and this seems to be an intrinsic property of the thalamocortical loop (reviewed in Dinse et al., 1997). One potential significance of this phenomenon is that a single volley of afferent spikes sets up a response outlasting by several hundred times the duration of the original stimulus. This may constitute a mechanism for the persistence of a mental impression aroused by a sensory stimulus. Recent evidence of replay of hippocampal spike trains coinciding with thalamocortical spindles (Siapas and Wilson, 1998) gives further credence to this hypothesis.

\section{SENSORY NEUROPROSTHESES}

Cortical microstimulation has been proposed as a method to deliver sensory percepts to circumvent damaged sensory receptors or pathways. To achieve desired encoding of percepts, it is essential to better understand the neural response to cortical microstimulation.

Recent work (Butovas et al., 2006) has suggested that the inhibitory period seen following cortical microstimulation is caused primarily through the influence of $\mathrm{GABA}_{\mathrm{B}}$ receptors in the cortex. The evoked oscillations we observe, and our model, suggest that thalamic neurons play a significant role in the observed response. These results can be reconciled since the experiment used to infer the role of $\mathrm{GABA}_{\mathrm{B}}$ involved an intraperitonal administration of $\mathrm{GABA}_{\mathrm{B}}$ antagonist making it impossible to distinguish between the role of $\mathrm{GABA}_{\mathrm{B}}$ at the level of the cortex or thalamus. Further, previous research (Grenier et al., 1998) has shown that the reticular nucleus plays a leading role in the rebound excitation observed in the cortex. It is therefore essential to keep in mind the excitation of thalamic neurons by cortical microstimulation during the design of stimulation protocols.

Thalamic gating modulates sensory inputs based on the behavioral state of the animal. As shown in this work, this mechanism also modulates the neural response to cortical stimulation. Further, behavioral state dictates whether thalamic neurons are in the tonic or burst firing mode and the resultant response to cortical microstimulation. Therefore it may be essential, in future sensory neuroprostheses, to modify stimulation parameters based on the behavioral state or background neural activity of the user in order to deliver desired percepts.

\section{APPENDIX}

Equations governing the TC block of the model:

$$
\begin{aligned}
C \frac{\mathrm{d} V_{\mathrm{TC}}}{\mathrm{d} t}= & -I_{\mathrm{AMPA}}-I_{\mathrm{GABA}_{\mathrm{A}}}-I_{\mathrm{GABA}_{\mathrm{B}}}-g_{\mathrm{K} \text { leak }}\left(V_{\mathrm{TC}}-V_{\mathrm{K}}\right) \\
& -g_{\mathrm{Na} \text { leak }}\left(V_{\mathrm{TC}}-V_{\mathrm{Na}}\right)
\end{aligned}
$$

$C$ is the membrane capacitance $\left(1 \mu \mathrm{F} / \mathrm{cm}^{2}\right), V_{\mathrm{TC}}$ is the membrane potential of TC neurons, $I_{\mathrm{AMPA}}, I_{\mathrm{GABA}}$ represent synaptic conductances, $g_{\mathrm{K} \text { leak }}\left(0.02 \mathrm{mS} / \mathrm{cm}^{2}\right)$ and $g_{\mathrm{Na} \text { leak }}\left(0.01 \mathrm{mS} / \mathrm{cm}^{2}\right)$ represent potassium and sodium leak conductances, $V_{\mathrm{K}}(-100 \mathrm{mV})$ and $V_{\mathrm{Na}}$ $(-55 \mathrm{mV})$ represent the reversal potential of the leak currents. The synaptic currents for AMPA are given by:

$I_{\mathrm{AMPA}}=g_{\mathrm{AMPA}}\left(V_{\mathrm{TC}}-V_{\mathrm{AMPA}}\right)$

$g_{\text {AMPA }}$ denotes the synaptic conductance and $V_{\text {AMPA }}(0 \mathrm{mV})$ denotes the reversal potential of the synaptic current. Synaptic conductances were modeled by convolving incoming spike firing rate [pulse $(t)$ ] with a synaptic impulse response function $h_{\text {AMPA }}(t)$ :

$$
\begin{aligned}
& g_{\mathrm{AMPA}}(t)=\int_{-\infty}^{t} h_{\mathrm{AMPA}}(t-\tau) \text { pulse }_{\mathrm{PY}}(\tau) \mathrm{d} \tau \\
& h_{\mathrm{AMPA}}(t)=A_{\mathrm{AMPA}}\left(\mathrm{e}^{-a 1_{\mathrm{AMPA}} t}-\mathrm{e}^{-a 2_{\mathrm{AMPA}} t}\right)
\end{aligned}
$$


Similar equations were used to calculate synaptic currents due to $\mathrm{GABA}_{\mathrm{A}}$ and $\mathrm{GABA}_{\mathrm{B}}$. GABA postsynaptic currents were assumed to increase nonlinearly with the firing density of RE and IN neurons. The nonlinear activation function is of the form:

$$
B\left(\text { pulse }_{\mathrm{RE}}\right)=\frac{1}{1+\mathrm{e}^{\left(\text {pulse }_{\mathrm{RE}}-\theta\right) / \sigma}}
$$

Membrane potential was converted to pulse densities using a sigmoidal transfer function with an extra component to account for burst firing in thalamic neurons that occurs at hyperpolarized membrane potential levels.

$$
\begin{aligned}
& \text { pulse }_{\mathrm{TC}}=\frac{G_{s}}{1+\mathrm{e}^{\left(V_{\mathrm{TC}}-\theta_{s}\right) / \sigma_{S}}}+G_{\mathrm{TC}} m_{\mathrm{TC} i n f}\left(V_{\mathrm{TC}}\right) n_{\mathrm{TC}}\left(V_{\mathrm{TC}}\right) \\
& n_{\mathrm{TC}}\left(V_{\mathrm{TC}}\right)=\int_{-\infty}^{t} h_{n}(t-\tau) n_{\mathrm{TC} i n f}\left(V_{\mathrm{TC}}\right) \mathrm{d} \tau \\
& h_{n}(t)=N\left(\mathrm{e}^{-n_{1} t}-\mathrm{e}^{-n_{2} t}\right)
\end{aligned}
$$

$$
\begin{aligned}
m_{\mathrm{TC} i n f} & =\frac{1}{1+\mathrm{e}^{\left(V_{\mathrm{TC}}-\theta_{m}\right) / \sigma_{m}}} \\
n_{\mathrm{TC} i n f} & =\frac{1}{1+e^{\left(V_{\mathrm{TC}}-\theta_{n}\right) / \sigma_{n}}}
\end{aligned}
$$

Physiological parameters used in this model were obtained from Suffczynski et al. (2004) and Wang et al. (1995). The entire model (in MATLAB) is available from the corresponding author upon request.

\section{ACKNOWLEDGMENTS}

This work was supported in part by the National Academies Keck Futures Initiative, the Christopher and Dana Reeve Foundation and the MARCO Microelectronics Advanced Research Corporation. We would like to thank Sandra Truong and Maria Dadarlat for assistance in behavioral training of rats, John Long for assistance with implantation surgery and Yang Dan, Shih-Chieh Lin, and Natalia Caporale for discussions related to this work.

\section{REFERENCES}

Ahissar,E.,Derdikman, D., Hildesheim, R., Arieli, A., and Grinvald, A. (2003). Imaging spatiotemporal dynamics of surround inhibition in the barrels somatosensory cortex. J. Neurosci. 23, 3100-3105.

Bartley, H. S., and Bishop, G. H. (1932). The cortical response to stimulation of the optic nerve in the rabbit. Am. J. Physiol. 103, 159-172.

Butovas, S., Hormuzdi, S. G., Monyer, H., and Schwarz, C. (2006). Effects of electrically coupled inhibitory networks on local neuronal responses to intracortical microstimulation. J. Neurophysiol. 96, 1227-1236.

Butovas, S., and Schwarz, C. (2003). Spatiotemporal effects of microstimulation in rat neocortex: a parametric study using multielectrode recordings. J. Neurophysiol. 90, 3024-3039.

Castro-Alamancos, M. A. (2004). Dynamics of sensory thalamocortical synaptic networks during information processing states. Prog. Neurobiol. 74, 213-247.

Castro-Alamancos, M.A., and Oldford, E. (2002). Cortical sensory suppression during arousal is due to the activity-dependent depression of thalamocortical synapses. J. Physiol. 541, 319-331.

Chang,H.(1949). The repetitive discharges of cortico-thalamic reverberating circuit. J. Neurophysiol. 235-257.

Contreras, D., Destexhe, A., Sejnowski, T. J., and Steriade, M. (1997). Spatiotemporal patterns of spindle oscillations in cortex and thalamus. J. Neurosci. 17, 1179-1196.

Contreras, D., and Steriade, M. (1996). Spindle oscillations in cats: the role of corticothalamic feedback in a thalamically generated rhythm. $J$. Physiol. 490, 159-179.

Cotillon, N., and Edeline, J. (2000). Tone-evoked oscillations in the rat auditory cortex result from interactions between the thalamus and reticular nucleus. Eur. J. Neurosci. 12, 3637-3650.

Cotillon-Williams, N., and Edeline, J. (2003). Evoked oscillations in the thalamo-cortical auditory system are present in anesthetized but not in unanesthetized rats. J. Neurophysiol. 89, 1968-1984.

Cotillon-Williams, N., and Edeline, J. (2004). Evoked oscillations in unit recordings from the thalamo-cortical auditory system: an aspect of temporal processing or the reflection of hyperpolarized brain states? Acta Neurobiol. Exp. 64, 253-270.

Delaney, K. R., Gelperin, A., Fee, M. S., Flores, J. A., Gervais, R., Tank, D. W., and Kleinfeld, D. (1994). Waves and stimulus-modulated dynamics in an oscillating olfactory network. Proc. Natl. Acad. Sci. U.S.A. 91, 669-673.

Destexhe, A., McCornick, D., and Sejnowski, T. J. (1993). A model for 8-10 Hz spindling in interconnected thalamic relay and reticularis neurons. Biophys. J. 65, 2473-2477.

Dinse, H. R., Krüger, K., Akhavan, A. C., Spengler, F., Schöner, G., and Schreiner, C. E. (1997). Low-frequency oscillations of visual, auditory and somatosensory cortical neurons evoked by sensory stimulation. $J$. Psychophysiol. 26, 205-227.

Fanselow, E. E., and Nicolelis, M. A. L. (1999). Behavioral modulation of tactile responses in the rat somatosensory system. J. Neurosci. 19, 7603-7616.
Ferezou,I.,Haiss,F.,Gentet,L. J.,Aronoff, R. Weber, B., and Petersen, C.C. H. (2007). Spatiotemporal dynamics of cortical sensorimotor integration in behaving mice. Neuron 56, 907-923.

Fontanini, A., and Katz, D. B. (2005). 7 to $12 \mathrm{~Hz}$ activity in rat gustatory cortex reflects disengagement from a fluid self-administration task. J. Neurophysiol. 93, 2832-2840.

Freeman, W. J. (2000). Neurodynamics: An Exploration of Mesoscopic Brain Dynamics. London, Springer.

Friedman-Hill, S., Maldonado, P. E., and Gray, C. M. (2000). Dynamics of striate cortical activity in the alert macaque: I. Incidence and stimulusdependence of gamma-band neuronal oscillations. Cereb. Cortex 10 1105-1116.

Fuentealba, P., and Steriade, M. (2005) The reticular nucleus revisited: intrinsic and network properties of a thalamic pacemaker. Prog. Neurobiol. 75, 125-141.

Fuentealba, P., Timofeev, I., and Steriade, M. (2004). Prolonged hyperpolarizing potentials precede spindle oscillations in the thalamic reticular nucleus. Proc. Natl. Acad. Sci. U.S.A. 101, 9816-9821.

Galambos, R., Rose, J. E., Bromiley, R. B., and Hughes, J. R. (1952). Microelectroded studies on medial geniculate body of cat. II. Response to clicks. J. Neurophysiol. 15, 359-380.

Gray, C. M., and Singer, W. (1989). Stimulus-specific neuronal oscillations in orientation columns of cat visual cortex. Proc. Natl. Acad. Sci. U.S.A. 86, 1698-1702.

Grenier, F., Timofeev, I., and Steriade, M (1998). Leading role of thalamic over cortical neurons during postinhibitory rebound excitation. Proc. Natl. Acad. Sci. U.S.A. 95, 13929-13934.

Hentschke, H., Haiss, F., and Schwarz, C. (2006). Central signals rapidly switch tactile processing in rat barrel cortex during whisker movements. Cereb. Cortex 16, 1142-1156.

Jagadeesh, B., Gray, C. M., and Ferster, D. (1992). Visually evoked oscillations of membrane potential in cells of cat visual cortex. Science 257, 552-554.

Kandel, A., and Buzsaki, G. (1997). Cellular-synaptic generation of sleep spindles, spike-and-wave discharges, and evoked thalamocortical responses in the neocortex of the rat. J. Neurosci. 17, 6783-6797.

Kelly, K.M. (2004). Spike-wave discharges: absence or not, a common finding in common laboratory rats. Epilepsy Curr. 4, 176-177.

Khazipov, R., Sirota, A., Leinekugel, X., Holmes, G. L., Ben-Ari, Y., and Buzsáki, G. (2004). Early motor activity drives spindle bursts in the developing somatosensory cortex. Nature 432, 758-761.

Lee, K. H., and McCormick, D. A. (1996). Abolition of spindle oscillations by serotonin and norepinephrine in the ferret lateral geniculate and perigeniculate nuclei in vitro. Neuron 17, 309-321.

Lee, S., Carvell, G. E., and Simons, D. J. (2008). Motor modulation of afferent somatosensory circuits. Nat. Neurosci. 11, 1430-1438.

Llinas, R. R., and Steriade, M. (2006). Bursting of thalamic neurons and states of vigilance. J. Neurophysiol. 95, 3297-3308.

McCormick, D. A., and Bal, T. (1997). Sleep and arousal: thalamocortical mechanisms. Annu. Rev. Neurosci. 20, 185-215. 
McCornick, D. (1992). Neurotransmitter actions in the thalamus and cerebral cortex and their role in neuromodulation of thalamocortical activity. Prog. Neurobiol. 39, 335-388.

Muthuswamy, J., Tran, P., Rangarajan, R., Lenz, F. A., Hanley, D. F., and Thakor, N. V. (1999). Somatosensory stimulus entrains spindle oscillations in the thalamic VPL nucleus in barbiturate anesthetized rats. Neurosci. Lett. 262, 191-194.

Nicolelis, M. A. L., and Fanselow, E. E. (2002). Thalamcortical optimization of tactile processing according to behavioral state. Nat. Neurosci. 5, 517-524.

Sally, S., and Kelly, J. B. (1988). Organization of auditory cortex in the albino rat: sound frequency. $J$. Neurophysiol. 59, 1627-1638.

Scaglione, A., and Moxon, K. (2006). Behaviorally modulated filter model for the thalamic reticular nucleus.
Conf. Proc. IEEE Eng. Med. Biol. Soc. 595-598.

Shaw, F. (2007). 7-12 Hz high-voltage rhythmic spike discharges in rats evaluated by antiepileptic drugs and flicker stimulation. J. Neurophysiol. 97, 238-247.

Sherman, S. (2001). Tonic and burst firing: dual modes of thalamocortical relay. Trends Neurosci. 24, 122-126.

Shin, H., and Chapin, J. (1990). Movement induced modulation of afferent transmission to single neurons in the ventroposterior thalamus and somatosensory cortex in rat. Exp. Brain Res. 81, 515-522.

Siapas, A. G., and Wilson, M. A. (1998). Coordinated interactions between hippocampal ripples and cortical spindles during slow-wave sleep. Neuron 21, 1123-1128.

Steriade, M., Jones, E. G., and Llinas, R. (1990). Thalamic oscillations and signaling. New York, John Wiley \& Sons.
Suffczynski, P., Kalitzin, S., and Lopes Da Silva, F. (2004). Dynamics of non-convulsive epileptic phenomena modeled by a bistable neuronal network. J. Neurosci. 126, 467-484.

Tehovnik, E. J., Tolias, A. S., Sultan, F., Slocum, W. M., and Logothetis, N. K. (2006). Direct and indirect activation of cortical neurons by electrical microstimulation. J. Neurophysiol. 96, 512-521.

Venkatraman, S. Elkabany, K., Long, J. D. Yao, Y., and Carmena, J. M. (2009). A system for neural recording and closedloop intracortical microstimulation in awake rodents. IEEE Trans. Biomed. Eng. 56, 15-22.

Wang, X. J., Golomb, D., and Rinzel, J. (1995). Emergent spindle oscillations and intermittent burst firing in a thalamic model: specific neuronal mechanisms. Proc. Natl. Acad. Sci. U.S.A. 92, 5577-5581.
Conflict of Interest Statement: The authors declare that the research was conducted in the absence of any commercial or financial relationships that could be construed as a potential conflict of interest.

Received: 03 March 2009; paper pending published: 06 April 2009; accepted: 15 May 2009; published online: 01 June 2009.

Citation: Venkatraman Sand Carmena JM (2009) Behavioral modulation of stimulusevoked oscillations in barrel cortex of alert rats. Front. Integr. Neurosci. (2009) 3:10. doi:10.3389/neuro.07.010.2009

Copyright (c) 2009 Venkatraman and Carmena. This is an open-access article subject to an exclusive license agreement between the authors and the Frontiers Research Foundation, which permits unrestricted use, distribution, and reproduction in any medium, provided the original authors and source are credited. 